\title{
Decoding 3D Disk Structure and Dynamics Using Doppler Tomography
}

\author{
Arthur D. Eigenbrot and Matthew A. Bershady \\ Department of Astronomy, University of Wisconsin - Madison, \\ 475 N. Charter St. Madison, WI, 53703, USA \\ email: eigenbrot@astro.wisc.edu
}

\begin{abstract}
We demonstrate a method to measure both rotation curves and 3D ISM structure in edge-on galaxies. Two-dimensional spectral coverage of edge-on galaxies reveals substantial deviations in emission line shapes from a purely gaussian profile that vary with radius and height. Non-gaussianity is quantified using statistical moments to third order. We infer the 3D density distribution by comparing the measured line profiles to synthetic line-of-sight velocity distributions from a suite of three-dimensional galaxy models with different 3D distributions of dust and gas and different rotation curve shapes and amplitudes. We apply this method using multi-position longslit data of nearby edge-on galaxy ESO 435-G25 and find our derived rotation curve matches measured HI rotation from envelope fitting but requires a flared dust disk to accurately describe the radial and vertical trends in the measured statistical moments. Our results are consistent with dynamical expectations for constant pressure support in a disk with exponentially declining surface-density.
\end{abstract}

\section{Conclusions}

Using optical longslit data from SALT we derive a rotation curve consistent HI envelope fitting (Kregel \& van der Kruit 2004) despite the effects of extinction and line-of-sight projection. We parametrize rotation by $V(r)=V_{\text {rot }} \tanh \left(r / h_{\text {rot }}\right)$ and find derived values of $h_{\text {rot }}$ and $V_{\text {rot }}$ that are consistent with the results of Andersen \& Bershady (2013), implying constant pressure support in an exponentially declining disk surface-density. We acknowledge support from NSF/AST-1009471
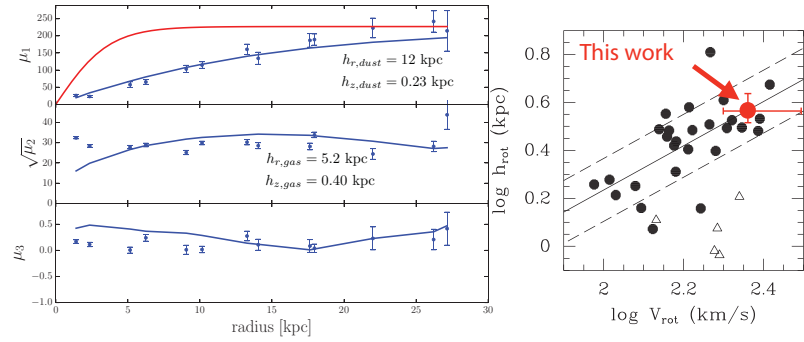

Figure 1. (left) The first three statistical moments as a function of radius for data from SALT (points) and the best fitting galaxy model (lines). The model rotation curve is shown as the red curve in the top panel. (right) Results from Andersen \& Bershady (2013) showing dynamical parameters $h_{\text {rot }}$ and $V_{\text {rot }}$ for a sample of disk galaxies. ESO 435-G25 is shown as the red point.

\section{References}

Andersen, D. R. \& Bershady, M. A. 2013, ApJ, 768

Andersen, D. R., et al. 2006, ApJS, 166

Kobulnicky, H. A., et al. 2002, Proceedings of SPIE, 4841, 1634

Kregel, M. \& van der Kruit, P. C. 2004 MNRAS, 352, 787-803

Xilouris, E. M., Byun, Y. I., Kylafis, N. D., Paleologou, E. V., \& Papamastorakis, J. 1999, A $\& A$, 34 